Josep Mensa ${ }^{*}$

Carlos Dueñas Gutiérrez ${ }^{2 *}$

Celia Cardozo ${ }^{1}$

Laura Rodríguez Fernández ${ }^{2}$

Martha Kestler $3,4,5,6$

Patricia Muñoz $3,4,5,6$

Emilio Bouza ${ }^{3,4,5,6}$

\title{
Neck infection after allogenic hematopoietic progenitors transplantation
}

${ }^{1}$ Infectious Disease Service, Hospital Clinic i Provincial. Barcelona. Spain.

${ }^{2}$ Infectious Disease Unit. Internal Medicine Service. Hospital Clínico Universitario de Valladolid. Spain.

${ }^{3}$ Division of Clinical Microbiology and infectious Diseases. Hospital General Universitario Gregorio Marañón, Madrid, Spain.

${ }^{4}$ Instituto de Investigación Sanitaria Gregorio Marañón, Madrid, Spain.

${ }^{5}$ Department of Medicine, School of Medicine, Universidad Complutense de Madrid (UCM), Spain.

${ }^{6}$ CIBER de Enfermedades Respiratorias (CIBERES CB06/06/0058), Madrid, Spain

\section{Article history}

Received: 5 December 2019; Revision Requested: 16 January 2020; Revision Received: 21 January 2020; Accepted: 28 January 2020; Published: 14 February 2020

\section{PRESENTATION OF CASE (DR. JOSEP. MENSA)}

A 65-year-old woman, diagnosed with Acute Myeloid Leukemia (AML) in December 2015, electively enters the Hematology Service in August 2016, to receive an allogeneic hematopoietic cell transplantation (ALLO-HCT), of peripheral blood, from an unrelated donor with an HLA 10/10 identity.

The transplant is performed after conditioning with fludarabine and busulfan. She received cyclophosphamide as prophylaxis for graft-versus host disease (GVHD) and subsequently maintained immunosuppression with tacrolimus and mycophenolate. Donor and recipient were seropositive for CMV and the patient received anti-infectious prophylaxis with levofloxacin $(500 \mathrm{mg} / \mathrm{d})$, fluconazole $(400 \mathrm{mg} / \mathrm{d})$ and acyclovir (800 mg/12h).

In the immediate post-transplant period, she presented grade IV mucositis and required parenteral nutrition and analgesia with opioids.

On the 5th post-transplant day, fever appeared, with no apparent focus, and empirical antibiotic treatment with meropenem was initiated ( $1 \mathrm{~g} / 8 \mathrm{~h}$ in $4 \mathrm{~h}$ extended perfusion).

Cervical bulk and cardio-respiratory arrest. On the 12th postransplant day, fever persisted and a right submandibular mass appeared. An Ear, Nose \& Throat (ENT) consul-

\footnotetext{
Correspondence:

Dr. Josep Mensa

Infectious Disease Service, Hospital Clinic I Provincial

Barcelona, Spain

E-mail:jmensa@icloud.com

Second corresponding author

Dr. Emilio Bouza

Instituto de Investigación Sanitaria Gregorio Marañón

C/ Dr. Esquerdo, 46

28007 Madrid, Spain

Phone: +34- 91- 3721721/Fax: +34-91- 5044906

E-mail: emilio.bouza@gmail.com

*Both to be considered first authors
}

tation suggested a probable sialoadenitis and daptomycin (10 $\mathrm{mg} / \mathrm{kg} /$ day) was added to the treatment with meropenem. Some blood test data at that time were as follows: PCR 18 $\mathrm{mg} / \mathrm{dL}$; Glucose $189 \mathrm{mg} / \mathrm{dL}$; Creatinine $0.76 \mathrm{mg} / \mathrm{dL}$; Glomerular Filtration $83 \mathrm{ml} / \mathrm{min}$; ASAT 73 IU/L; ALAT 34 IU/L; GGT 107 IU/L; Total Bilirubin $0.9 \mathrm{mg} / \mathrm{dL}$; Direct bilirubin $0.4 \mathrm{mg} / \mathrm{dL} ; A \mathrm{~A}-$ kaline Phosphatase $94 \mathrm{IU} / \mathrm{L} ; \mathrm{LDH} 614 \mathrm{IU} / \mathrm{L} ; \mathrm{Na} 130 \mathrm{mEq} / \mathrm{L} ; \mathrm{K}$ $3.1 \mathrm{mEq} / \mathrm{L}$; Leukocytes $0.04 \times 10^{9} / \mathrm{L} ; \mathrm{Hb} 9.3 \mathrm{~g} / \mathrm{dL}$; Platelet count $38 \times 10^{9} / \mathrm{L}$. Ferritin $1,080 \mathrm{ng} / \mathrm{mL}$ and sideremia $106 \mu \mathrm{g} / \mathrm{dL}$. Blood cultures, urine culture and serum Aspergillus Galactomannan Assay (AGA) determinations were negative. Cytomegalovirus (CMV) blood viral load was undetectable in 3 determinations.

In the next 24 hours, the inflammation spreaded through the laterocervical and parotid region and, in the early morning of the 14th post-transplant day, the patient developed a rapidly progressive respiratory difficulty with costal tirage and laryngeal stridor. She was transferred to the emergency operative room where she was admitted with a 3 Glasgow Coma Score and agonic respiration, so advanced Cardio-Pulmonary Resuscitation was initiated. Oro-Tracheal intubation was unsuccessfully attempted under direct vision and with glidescope. Manual ventilation with bag-mask was performed while the ENT surgeon performed an successful emergent tracheostomy, with patient's recovery of pulse and oxygen saturation. Approximate time without effective ventilation was calculated in about $10 \mathrm{~min}$.

Given the thrombocytopenia and the possibility that the abrupt deterioration was due to a hemorrhagic complication, a continuous perfusion of platelets was maintained during the operation and in the following 24 hours.

ICU admission. The patient was admitted to the Intensive Care Unit (ICU), where on admission macroglossia was observed, making it impossible to assess the situation of the oral cavity. A large indurated mass was palpated in the right latero-cervical and submandibular region. The facies was edematous and there was epistaxis, contained with anterior nasal packing. 


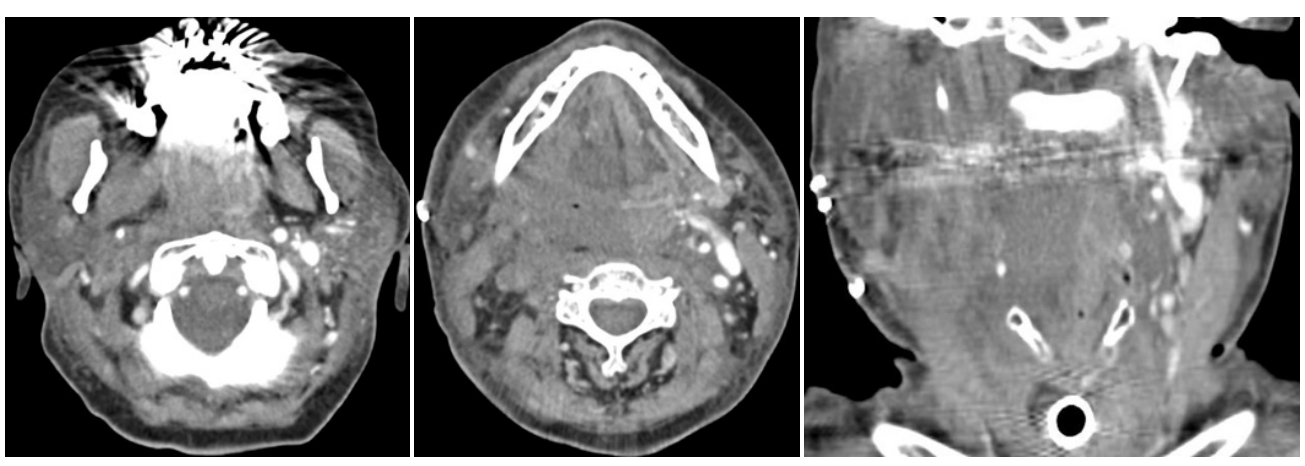

\begin{tabular}{l|l} 
Figure 1 & Computed tomography scan. Day + 14 after transplantation
\end{tabular}

Some analytical data in blood were at that moment as follows: PCR 29 mg/dL, Glucose 158 mg/dL, Cr $1.17 \mathrm{mg} / \mathrm{dL}$, Glomerular Filtrate $48 \mathrm{ml} / \mathrm{min}$, ASAT $121 \mathrm{UI} / \mathrm{L}$, ALAT $43 \mathrm{UI} / \mathrm{L}$, GGT $132 \mathrm{UI} / \mathrm{L}$, Total Bilirubin $2.6 \mathrm{mg} / \mathrm{dL}$, Alkaline Phosphatase 60 UI/L, LDH $826 \mathrm{UI} / \mathrm{L}$, Sodium $136 \mathrm{mEq} / \mathrm{L}$, Potassium $2.6 \mathrm{mEq} / \mathrm{L}$. Leukocytes $0.07 \times 10^{9} / \mathrm{L}$, hemoglobin $13.7 \mathrm{~g} / \mathrm{dL}$, HT 37\%, MCV $87.9 \mathrm{fl}$, platelets $24,000 / \mathrm{uL}$. Hemostasis with Prothrombin Time of 80\% and activated Partial Thromboplastin Time (aPT) of 40 $\mathrm{sec}$, with international normalized ratio (INR) of 1.13. Blood cultures were negative and after post-surgical stabilization, cervical computed tomography (CT) was performed (figure 1).

The CT scan was reported as follows: "Occupation of soft tissue extending from the nasopharynx to the trachea, completely collapsing the airway. Well positioned permeable endotracheal tube. Thickening of the cervical subcutaneous cellular tissue and right predominant platysma muscle. Extensive ill-defined hypodense area with loss of differentiation of the structures of the floor of the mouth affecting the muscles of the sublingual space with hypocaptation of the right submaxillary gland with some gas bubble inside and at the level of vallecules.

The right deep cervical planes are also hypodense with a puffy aspect with loss of differentiation of the parapharyngeal, retropharyngeal, carotid and prevertebral spaces on the right side with extension of the phlegmonous process towards the contralateral parapharyngeal and vascular space.

Internal jugular and right common carotid do not appear opacified and therefore thrombosis cannot be ruled out. Mucosal occupation of maxillary, ethmoidal and sphenoidal paranasal sinuses".

Treatment with meropenem $(2 \mathrm{~g} / 8 \mathrm{~h})$, daptomycin (10 $\mathrm{mg} / \mathrm{kg} / 24 \mathrm{~h}$ ) and clindamycin (600 mg/8 h) was maintained. Bleeding persisted around the tracheostoma and oral cavity. Continuous platelet perfusion was maintained for the next 48 hours until the figure of 80,000 platelets/uL was achieved.

Sedo-analgesia was discontinued for neurological assessment. No response to stimuli and persistence of low level of consciousness was observed. Cranial CT scan was performed, showing no significant findings.
EEG showed signs compatible with severe anoxic encephalopathy.

Forty-eight hours later, right arreactive anisocoria was objective. In a new cranial CT scan, ischemic stroke was observed in the territory of the right posterior inferior cerebellar artery (PICA). In this context, she presented hemodynamic instability requiring vasoactive drugs to maintain Mean Arterial Pressure around $65 \mathrm{mmHg}$.

Evoked potentials showed absence of bilateral cortical evoked response by stimulating both medium nerves.

After informing the family of the poor life prognosis, it was decided to prioritize the patient's comfort measures and limitation of therapeutic effort. The patient was deceased and a necropsy was performed.

\section{DIFFERENTIAL DIAGNOSIS OF THE PATIENT (DR. CARLOS DUEÑAS)}

The circumstances that contribute to the etiology of the infection in this patient are those accumulated by the basic pathology of the recipient, in this case $A M L$, those derived from the invasive procedure (in this case practically null) and those related to immunosuppressive treatment (in this case busulfan, fludarabine, cyclophosphamide, mycophenolate and tacrolimus).

AML is the haematological disease with the highest risk of Invasive Fungal Infections (IFIs) with an incidence that varies between 10 and 25 percent, according to studies by SEIFEM [1]. The risk factors for IFI in AML are classified in 4 categories (table 1)

Looking at it from the point of view of infections in ALLO-HCT there are also a number of factors that according to Wingard et al. [2]. influence its presentation (table 2).

One aspect that should be pointed out, after reviewing the risk factors involved in this patient, is that of iron overload. Several articles underline that in patients with ALLO-HCT, iron overload is a poor prognostic factor for survival [3-5].

In this patient, another aspect of interest is the poten- 


\begin{tabular}{l|l}
\hline Table 1 & $\begin{array}{l}\text { Risk factors for Invasive Fungal } \\
\text { Infections (IFIs) in Acute Myeloid } \\
\text { Leukemia (AML) }\end{array}$ \\
\hline a) Factors related to the Leukemia \\
Advanced stage of the disease \\
Complete remission failure \\
\hline b) Host-related factor \\
Comorbidities \\
Older age \\
Organ dysfunction \\
Unfavorable genetic compatibility \\
\hline c) Treatment related factors \\
Severity and duration of neutropenia (The duration of neutropenia is \\
10-14 days after autologous HCT and 15-30 days after Allo-HCT using \\
ablative therapy and 5-7 days if ablative therapy is not used) \\
Severe mucositis, associated with chemotherapy \\
\hline d) Factors related to exposure to fungi \\
Rooms without HEPA filter and previous IFls
\end{tabular}

tial role of the myeloablative regimen in the evolution of this case. The regimen consisting of busulfan and cyclophosphamide is considered the classical myeloablative treatment and is associated with earlier, longer-lasting toxicities and higher mortality, primarily in older people than treatment with busulfan and fludarabine [6]. Fludarabine is less toxic than cyclophosphamide, maintaining its immunosuppressive efficacy and the combination busulfan-fludarabine is associated with a lower risk of sinusoidal obstruction syndrome characterized by hyper-bilirrubinemia (> $2 \mathrm{mg} / \mathrm{dl}$ ), painful hepatomegaly and weight gain secondary to ascites. It is also associated with a lower risk of documented infections. The risk of grade 3-4 mucositis or graft rejection is similar with both drugs.

In order to discuss the possible etiology of this patient's disease, it is indicative to review the relationship that the infections have with the post-transplant moment. In the first 30 days after the procedure, the major problems are damage to the phagocytosis and the mucocutaneous barrier as a consequence of the treatment in preparation for the transplant. Prolonged neutropenia is added to this. As a consequence of the above, the main sources of infection will be the oropharyngeal, gastrointestinal and cutaneous flora.

Frequent use of intravenous catheters can serve as a gateway for opportunistic skin colonizing pathogens such as coagulase-negative staphylococci (CoNS), Staphylococcus aureus, Enterococcus spp. and Candida spp. In general, the focus of infection is not usually found and antibiotic treatment is usually empirical, covering bacterial infections. Colony stimulating growth factors are often used to decrease the duration of neutropenia and its complications, and in the case of persistent neutropenia is often associated with the appearance of Aspergillus spp. Reactivation of Herpes simplex virus (HSV) may also occur [7].

\begin{tabular}{|c|c|}
\hline Table 2 & $\begin{array}{l}\text { Factors influencing the risk of infections } \\
\text { in ALLO-HCT. }\end{array}$ \\
\hline \multicolumn{2}{|c|}{ PATIENT DEPENDENT FACTORS } \\
\hline \multicolumn{2}{|c|}{ Advanced age } \\
\hline \multicolumn{2}{|c|}{ High comorbidity index for ALLO-HCT } \\
\hline \multicolumn{2}{|c|}{ Factors related to underlying disease or previous treatments } \\
\hline \multicolumn{2}{|c|}{ (corticoids, cyclophosphamide) } \\
\hline \multicolumn{2}{|c|}{ Underlying diseases } \\
\hline \multicolumn{2}{|c|}{ Previous HCT transplant } \\
\hline \multicolumn{2}{|c|}{ Previous donor or recipient infections } \\
\hline \multicolumn{2}{|c|}{ Previous immunity for CMV, VHS, WZ, VEB } \\
\hline \multicolumn{2}{|c|}{ Iron overload } \\
\hline \multicolumn{2}{|c|}{ TRANSPLANT DEPENDENT FACTORS } \\
\hline \multicolumn{2}{|c|}{ Myeloablative regimens } \\
\hline \multicolumn{2}{|c|}{ Donate/receiver HLA compatibility: 10/10 } \\
\hline \multicolumn{2}{|c|}{ Type of cell transplant performed: umbilical cord or peripheral blood } \\
\hline \multicolumn{2}{|c|}{ T-cell deplection } \\
\hline \multicolumn{2}{|c|}{ Immunosuppressive Regime: Methotrexate } \\
\hline \multicolumn{2}{|c|}{ INMUNOGENETICS } \\
\hline \multicolumn{2}{|c|}{ Polymorphisms that increase or decrease the risk of infection } \\
\hline \multicolumn{2}{|c|}{ PROLONGED AND SEVERE NEUTROPENIA } \\
\hline \multicolumn{2}{|c|}{$\begin{array}{l}\text { MASCC risk index: (13 points in this case, representing a high risk of poor } \\
\text { prognosis) }\end{array}$} \\
\hline \multicolumn{2}{|c|}{$\begin{array}{l}\text { Acute or chronic and prolonged GVHD and its treatment: above all use } \\
\text { of high-dose corticosteroids and new immunosuppressants that delay } \\
\text { immune reconstitution. }\end{array}$} \\
\hline \multicolumn{2}{|c|}{ Infection with immunomodulatory virus: CMV } \\
\hline \multicolumn{2}{|c|}{ Graft failure } \\
\hline Respirat & nplications \\
\hline
\end{tabular}

Fever in the neutropenic transplant recipient is common in the pre-graft phase. Fever typically appears from the $3 \mathrm{rd}$ to the 5th day after the onset of neutropenia and may be the only symptomatology of the infection. The most frequent in this situation are bacterial infections, but in many cases microbiological documentation is not obtained due to early initiation of broad-spectrum antibiotic treatment. There are only positive blood cultures in 10-25\% of the cases. When foci of infection are identified (20-30\% of occasions) the most frequent origins are: the lungs, the skin, especially in the insertion zone of the catheter and the perianal area, the genitourinary tract, the oral cavity, and the gastrointestinal tract. In the absence of apparent focus, the standard antibiotic treatment is monotherapy with a broad-spectrum Beta-lactam drug, such as piperacillin/tazobactam, ceftazidime, cefepime or a carbapenem with broad Gram-negative spectrum of activity (BGNs) including $P$. aeruginosa.

In the presence of catheter-related infection, cellulitis, 
pneumonia, mucositis or methicillin-resistant S. aureus (MRSA) colonization, drugs with MRSA activity such as glycolypopeptides should be added.

Persistence of fever may be due to a delay in response to the initial regimen established, the existence of Gram-positive infection not treated with the initial antibiotic regimen, the presence of infection with BGNs resistant to the treatment established, the presence of untreated fungal infections or causes that are not infectious.

We would like to briefly discuss the value of acute phase reactants in this situation. The specificity of PCR is low for infections. It usually increases within 24 hours of infection and can predict the development of fever and sepsis in neutropenic patients. Procalcitonin (PCT) is more specific for the diagnosis of bacterial infections in febrile neutropenia with a very high NPV for bacteremia [8].

Our patient had mucositis. Mucositis serves as a gateway to microorganisms in the blood, such as bacteremia by $S$. viridans and CoNS. Oral bacterial microbiota usually changes after chemotherapy and increases in colonization by microorganisms such as Enterococcus faecalis and Candida spp. have been described in ALLO-HCT.

Searching for an etiologic diagnosis of the potential infection, our patient was receiving prophylaxis against viral infection. All recipients with positive CMV serology and all negative recipients with positive CMV donor antibodies, should receive ganciclovir prophylaxis from before transplantation until 100 days later. For HSV, acyclovir prophylaxis should be offered to all patients with positive serology to prevent reactivation in the early phase after grafting and maintain it until mucositis resolves and in the post-graft phase, approximately until 30 days after transplantation [9-11]. The prophylaxis with acyclovir and the repeated negativity of the CMV viral load in this patient, invite me to rule out the participation of both viruses in the case that we are discussing.

Prophylactic treatment of bacterial infection, in these circumstances, with fluoroquinolones, has a role in the prevention of early infection and there are studies indicating that the use of prophylactic antibiotics reduces the number of bacteremias, without reducing the associated mortality [12]. In contrast, the use of fluoroquinolones is associated with the development of quinolone-resistant CoNS infections, Streptococcus of the viridans group, and E. coli. Levofloxacin is preferred over ciprofloxacin because of its better coverage against $S$. viridans. The use of vancomycin is associated with the appearance of vancomycin-intermediate $S$. aureus and vancomycin-resistant Enterococcus [13] and the use of fluoroquinolones is associated with the appearance of $\mathrm{BGN}$ infections resistant to them [14].

In our opinion, in this case, the bacterial infection, due to intense antibiotic treatment, is either not present or is due to the presence of MDR microorganisms not treatable with the multiple antimicrobials administered.

Regarding fungal infections, this patient received proph- ylaxis with fluconazole, which decreases the risk of invasive candidiasis during the neutropenic phase, especially in centers where Candida albicans is the main cause of infections in the pre-graft phase. The problem is that fluconazole is not active against certain Candida species such as $C$. krusei or a significant percentage of $C$. glabrata, nor against filamentous fungi. Prophylactic strategies have led to a decrease in systemic $C$. albicans infections, although esophageal infection often persists in these cases. C. glabrata and C. kefyr are associated with the presence of oral ulcers in TCH. Filamentous fungi such as Aspergillus and any of the mucorales may produce lesions that mimic mucositis [15].

The prevalence of invasive fungal infection demonstrated in autopsies during the last 2 decades in these patients oscillates around 31\%, and most of them are a necropsic finding. The most frequently isolated fungi are Candida spp. and Aspergillus spp. and, less frequently, Fusarium, Scedosporium, Mucorales and other emerging fungi, probably related to the selection of species produced with the use of the new antifungals. Predisposing factors are: advanced age, type of underlying haematological disease, immunosuppression, administration of broad-spectrum antibiotics, use of central venous catheter (CVC) for long periods of time, administration of parenteral nutrition, malnutrition, alteration of anatomical barriers (skin and mucous membranes), prolonged neutropenia, use of corticoids in rejection, iron overload and presence of genetic polymorphisms TLR-4, dectin 1 and pentraxin. The absence of Candida isolates in any sample or time and the prophylaxis with fluconazole invite me to put Candida infection in a very secondary place of preference as the cause of this patient's process.

Among the filamentous fungi, Aspergillus has a particular importance. Most cases are caused by Aspergillus fumigatus and are followed in frequency by $A$. flavus, $A$. terreus, $A$. niger and $A$. glaucus. The incidence can reach $8 \%$ in TPH and the most relevant risk factors are: prolonged neutropenia, intensity of immunosuppressive treatment, treatment with corticoids and concomitant viral infections by CMV and respiratory syncytial virus (RSV). Diagnosis of invasive aspergillosis is difficult because there are no characteristic clinical signs, imaging tests are not always conclusive, and laboratory methods have little sensitivity. Biomarkers such as galactomannan antigen have variable sensitivity and specificity in transplant recipients, depending on the type of sample, frequency of the sample, and the type of antigen, sampling and interpretive cut-off points. The greatest experience and usefulness has been demonstrated in granulocytopenic $\mathrm{TPH}_{\text {, }}$ with positive and negative predictive values of 94.4 and $98 \%$, respectively, preceding the appearance of symptoms in up to $80 \%$ of patients. False positive results associated with various biological factors are also known, such as colonization by Bifidobacterium, absorption of food galactomannan in patients with chronic GVHD, treatment with old piperacillin-tazobactam or amoxicillin-clavulanic preparations, and cross-reactivity with other fungi such as Penicillium, Alternaria or Paecilomyces [16]. In this patient, Aspergillus has 
not been isolated at any time, there is no lung infection and galactomannan is reported as repeatedly negative, making the diagnosis of invasive aspergillosis unlikely.

Following with other filamentous fungi, all deep infections caused by Scedosporium, Fusarium and Mucoraceae are very serious and potentially fatal $[17,18]$. Good evolution, when occurs, is usually related to a quick diagnosis (by direct microscopic vision of the samples of biopsied lesions), early antifungal treatment (often combining two or more drugs), extensive surgical debridment of the accessible lesions and the recovery of the patient's immune status, especially the neutropenia.

Fusarium incidence has increased due to the increased use of cytostatic treatment and ALLO-HCT and has been associated with soft tissue infections, onychomycosis and keratitis in immunocompromised patients from where it can disseminate. Inhalation of spores is another portal of entry producing sinusitis and pneumonia. Nucci et al. [19]. described a trimodal distribution of fusariosis in allo-HCT: a first peak before neutropenia recovery, a second peak at 62 days of mean transplant and a third peak after one year of transplantation. It usually presents with persistent fever and very pleomorphic skin metastatic lesions (nodules, ulcers) that can evolve towards central necrosis. Galactomannan can be useful for diagnosis and blood cultures are usually positive up to 50\% of the time. There are no skin lesions in this patient, the presentation is like a cervical mass in the neck, galactomannan is negative and we are not informed of any positive blood cultures, so we believe that Fusarium is very unlikely to cause this picture.

Scedosporium, is another gender to consider in this disease. It can be a filamentous fungui of very aggressive behavior in neutropenic patients. Scedosporium apiospermum is angioinvasive and can cause sinopulmonary affection, endophthalmitis and dissemination to the central nervous system (CNS). Scedosporium prolificans is a dematiaceous fungus, phylogenetically close to the genus Petriella which causes disseminated infections with high mortality due to its special virulence and resistance to almost all available antifungals. Curiously, most of the IFls by S. prolificans have been described in the Iberian Peninsula, California, United Kingdom and Australia $[20,21]$. It is not possible to find information of Scedosporium infections with neck soft tissue invasion similar to this patient. It is a cause, however of sinusitis and otomycosis [22-26] which makes it unlikely that this fungus is the causal agent in this case.

The most frequent agents of mucormycosis are fungi of the genera Rhizopus, Mucor or Lichtheimia and risk factors for its appearance include prolonged neutropenia, treatment with corticoids, diabetic ketoacidosis and iron overload, many of which are present in this patient. Mucormycosis in ALLO-HCT usually appears early in the graft or later, as a complication of graft rejection. It is usually manifested as a rhinocerebral or pulmonary disease. In the rhinocerebral form the most frequent presentation is fever, facial pain and headache. It can present extension into contiguous spaces towards the orbit, palate or brain and is the first cause of invasive sinusitis in haematological patients. Its speed to invade tissues and spread through blood vessels (angioinvasion) is one of the causes of the high mortality rate (> 90\%).

Mucormycosis can cause tissue invasion in the neck and mimic Ludwig's Angina [27].

Regarding the ferric overload in this disease, iron is an essential element for the growth and virulence of most microorganisms. The states of iron overload increase the risk of IFI by increasing the concentration of free iron, a necessary element for fungal growth and for the development of its mechanisms. Ferric overload and the consequent increase in the concentration of free iron are risk factors for the development of IFI by Mucoraceae. During ALLO-TCH pre-conditioning the IST can reach $100 \%$ on the second day with the consequent increase in iron not linked to transferrin. Mucoraceae are more dependent on the availability of free iron in the medium than Aspergillus, as this genus has more efficient mechanisms for its uptake from serum transferrin. Therefore, due to the rapid progression of the disease and the factors above mentioned, it cannot be excluded a mucormycosis as the final cause of death of this disease. The rapid progression of the neck lesion could be attributed to a suppurative septic thrombophlebitis of the jugular. This complication of neck infections is a multisystemic infection with septic emboli and possible thrombotic extension to the CNS [28].

Finally, we consider the parasitic aetiology of this picture unlikely. It is not known the serological status of this patient against Toxoplasma but anti-toxoplasma prophylaxis should be evaluated in case of positive serology recipient with acute graft rejection or history of toxoplasmic chorioretinitis, in principle with TMP-SMX [29, 30].

From all of the above, it is believed that this patient does not have a viral or parasitic infection, bacterial infection is unlikely, and we are inclined to think that it is a fungal infection. Of these, mucormycosis seems to be the most compatible with the clinical picture but it is not possible to rule out other filamentous fungi such as Fusarium, Scedosporium or dematiaceous fungi.

Infection with multi-resistant Gram-negative bacilli should be contemplated but seems highly unlikely in this disease.

\section{EVOLUTION OF THE PATIENT (DR. JOSEP MENSA)}

The summary of the autopsy report reads: Angioinvasive Mycosis disseminated by Mucor sp. with involvement of the cervical area with necrotic tumour that exceeds the midline and compresses the upper airway. Subglottic hematoma covered by laryngeal mucosa.

- Extensive cerebral vascular involvement with thrombosis by fungal stuctures and parenchymatous infarction (area of the right posteroinferior cerebellar artery and the territory of the left temporal lobe).

- Extensive fungal invasion of the arteries of the gastric 
submucosa with phenomena of ischemic necrosis of the gastric wall in the body and fundus.

- Multiple fungal pulmonary thromboembolism with thrombus in distal LII and proximal LSI arteries. Extensive ischemic-type pulmonary infarcts with fungal overgrowth. Fungal thrombosis of the splenic vein. Extensive coagulative necrosis in the tail of the pancreas, peripancreatic fat and splenic parenchyma. Septic emboli in adrenal fat with secondary fat necrosis.

\section{DISCUSSION OF CASE}

Lanternier and colleagues reviewed 101 cases of mucormycosis (60 tested, 41 probable) in France, 50\% of them with haematological malignancy. The episodes occurred in an average time after the onset of the disease of 8.8 months. The authors established an estimated incidence of mucormycosis in organ and stem cell transplant recipients of 8\% [31].

The involvement of the mouth's floor and the upper part of the neck as a presentation of mucormycosis is uncommon but well described $[27,32]$. Surgery is an essential component of the therapeutic strategy because antifungal treatment alone is often ineffective in controlling infection. In a series of 49 patients with rhinocerebral mucormycosis, mortality was $70 \%$ in cases treated with antifungals alone versus 14\% treated with antifungals and surgery [33].

The antimicrobial treatment of rhinocerebral mucormycosis is the association of amphotericin B with an echinocandin that may be synergistic [34]. Rhizopus oryzae expresses the gene that encodes the proteins of the 1,3-b-D-glucan synthase complex, whose activity is inhibited by caspofungin. At a dose of $0.5 \mathrm{mg} / \mathrm{kg}$ every $12 \mathrm{~h}$ caspofungin improved survival in a model of mucormycosis in diabetic rats [35].

\section{FINAL DIAGNOSIS}

Angioinvasive mycosis disseminated by Mucor sp. with involvement of the cervical area.

\section{REFERENCES}

1. Pagano L, Busca A, Candoni A, Cattaneo C, Cesaro S, Fanci R, et al. Risk stratification for invasive fungal infections in patients with hematological malignancies: SEIFEM recommendations. Blood Rev. 2017;31(2):17-29. PMID:27682882

2. Wingard JR, Hsu J, Hiemenz JW. Hematopoietic stem cell transplantation: an overview of infection risks and epidemiology. Infect Dis Clin North Am. 2010;24(2):257-72. PMID:20466269

3. Sivgin S, Baldane S, Deniz K, Zararsiz G, Kaynar L, Cetin M, et al. Increased Hepatic Iron Content Predicts Poor Survival in Patients With Iron Overload Who Underwent Allogeneic Hematopoietic Stem Cell Transplantation. Clin Lymphoma Myeloma Leuk. 2016;16 Suppl:S10-8. PMID:27521305

4. Sivgin S, Baldane S, Kaynar L, Kurnaz F, Pala C, Ozturk A, et al. Pretransplant serum ferritin level may be a predictive marker for outcomes in patients having undergone allogeneic hematopoietic stem cell transplantation. Neoplasma. 2012;59(2):183-90. PMID:22248276

5. Sucak GT, Yegin ZA, Ozkurt ZN, Aki SZ, Yagci M. Iron overload: predictor of adverse outcome in hematopoietic stem cell transplantation. Transplant Proc. 2010;42(5):1841-8. PMID:20620535

6. Ben-Barouch S, Cohen O, Vidal L, Avivi I, Ram R. Busulfan fludarabine vs busulfan cyclophosphamide as a preparative regimen before allogeneic hematopoietic cell transplantation: systematic review and meta-analysis. Bone Marrow Transplant. 2016;51(2):232-40. PMID:26457908

7. Centers for Disease Control and Prevention, Infectious Diseases Society of America, American Society of Blood and Marrow Transplantation. Guidelines for preventing opportunistic infections among hematopoietic stem cell transplant recipients. MMWR Recomm Rep. 2000;49(RR-10):1-125, CE1-7. PMID: 11718124

8. Mori Y, Miyawaki K, Kato K, Takenaka K, Iwasaki H, Harada N, et al. Diagnostic value of serum procalcitonin and $\mathrm{C}$-reactive protein for infections after allogeneic hematopoietic stem cell transplantation versus nontransplant setting. Intern Med. 2011;50(19):2149-55. PMID:21963733

9. Saral R, Burns WH, Laskin OL, Santos GW, Lietman PS. Acyclovir prophylaxis of herpes-simplex-virus infections. N Engl J Med. 1981;305(2):63-7. PMID:6264292

10. Gluckman E, Lotsberg J, Devergie A, Zhao XM, Melo R, Gomez-Morales $M_{1}$ et al. Prophylaxis of herpes infections after bone-marrow transplantation by oral acyclovir. Lancet. 1983;2(8352):706-8. PMID:6136841

11. Wade JC, Newton B, McLaren C, Flournoy N, Keeney RE, Meyers JD. Intravenous acyclovir to treat mucocutaneous herpes simplex virus infection after marrow transplantation: a double-blind trial. Ann Intern Med. 1982;96(3):265-9. PMID:7036816

12. Cruciani M, Rampazzo $R$, Malena M, Lazzarini L, Todeschini G, Messori $A$, et al. Prophylaxis with fluoroquinolones for bacterial infections in neutropenic patients: a meta-analysis. Clin Infect Dis. 1996;23(4):795-805. PMID:8909847

13. Seo SK, Xiao K, Huang YT, Jongwutiwes U, Chung D, Maloy M, et al. Impact of peri-transplant vancomycin and fluoroquinolone administration on rates of bacteremia in allogeneic hematopoietic stem cell transplant (HSCT) recipients: a 12-year single institution study. J Infect. 2014;69(4):341-51. PMID:24931578

14. Miles-Jay A, Butler-Wu S, Rowhani-Rahbar A, Pergam SA. Incidence rate of fluoroquinolone-resistant gram-negative rod bacteremia among allogeneic hematopoietic cell transplantation patients during an era of levofloxacin prophylaxis. Biol Blood Marrow Transplant. 2015;21(3):539-45. PMID:25498393

15. Haverman TM, Raber-Durlacher JE, Rademacher WM, Vokurka S,

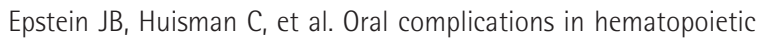
stem cell recipients: the role of inflammation. Mediators Inflamm. 2014;2014:378281. PMID:24817792

16. Perez JL, Ayats J, Fortun J, de Ona M, Pumarola T. Microbiología del trasplante. Enferm Infecc Microbiol Clin. 2011;29(9):683-90. PMID:21726920 
17. Nucci M, Marr KA, Queiroz-Telles F, Martins CA, Trabasso P, Costa $S$, et al. Fusarium infection in hematopoietic stem cell transplant recipients. Clin Infect Dis. 2004;38(9):1237-42. PMID:15127334

18. Peman J, Salavert M. Enfermedad fungica invasora por Scedosporium, Fusarium y Mucor. Rev Iberoam Micol. 2014;31(4):242-8. PMID:25442383

19. Nucci $M$, Anaissie E. Fusarium infections in immunocompromised patients. Clin Microbiol Rev. 2007;20(4):695-704. PMID:17934079

20. Berenguer J, Rodriguez-Tudela JL, Richard C, Alvarez M, Sanz MA, Gaztelurrutia L, et al. Deep infections caused by Scedosporium prolificans. A report on 16 cases in Spain and a review of the literature. Scedosporium Prolificans Spanish Study Group. Medicine (Baltimore). 1997;76(4):256-65. PMID:9279332

21. Castiglioni B, Sutton DA, Rinaldi MG, Fung J, Kusne S. Pseudallescheria boydii (Anamorph Scedosporium apiospermum). Infection in solid organ transplant recipients in a tertiary medical center and review of the literature. Medicine (Baltimore). 2002;81(5):33348. PMID:12352630

22. Baumgartner BJ, Rakita RM, Backous DD. Scedosporium apiospermum otomycosis. Am J Otolaryngol. 2007;28(4):254-6. PMID:17606042

23. Ference EH, Kubak BM, Zhang P, Suh JD. Successful Treatment of Scedosporium Sinusitis in Two Lung Transplant Recipients: Review of the Literature and Recommendations for Management. Allergy Rhinol (Providence). 2019;10:2152656719827253. PMID: 30792939

24. Khoueir N, Verillaud B, Herman P. Scedosporium apiospermum invasive sinusitis presenting as extradural abscess. Eur Ann Otorhinolaryngol Head Neck Dis. 2019;136(2):119-21. PMID:30528155

25. Kishimoto I, Shinohara S, Ueda T, Tani S, Yoshimura H, Imai Y. Orbital apex syndrome secondary to a fungal nasal septal abscess caused by Scedosporium apiospermum in a patient with uncontrolled diabetes: a case report. BMC Infect Dis. 2017;17(1):649. PMID:28950832

26. Salamat AA, Archer $C$, Basarab A, Eren E, Batty $V$, Patel $S$, et al. Scedosporium apiospermum causing otomycosis in an immunocompetent child with tympanostomy tubes: Management of this rare entity. Int J Pediatr Otorhinolaryngol. 2015;79(10):1785-7. PMID:26298623

27. McSpadden RP, Martin JR, Mehrotra S, Thorpe E. Mucormycosis Causing Ludwig Angina: A Unique Presentation. J Int Med Res. PMID: 30958072

28. Johannesen KM, Bodtger U. Lemierre's syndrome: current perspectives on diagnosis and management. Infect Drug Resist. 2016;9:221-7. PMID: 27695351

29. Foot $A B$, Garin $Y J$, Ribaud $P$, Devergie A, Derouin $F$, Gluckman E. Prophylaxis of toxoplasmosis infection with pyrimethamine/sulfadoxine (Fansidar) in bone marrow transplant recipients. Bone Marrow Transplant.. 1994;14(2):241-5. PMID:7994239

30. Peacock JE, Jr., Greven CM, Cruz JM, Hurd DD. Reactivation toxoplasmic retinochoroiditis in patients undergoing bone marrow transplantation: is there a role for chemoprophylaxis? Bone Marrow Transplant. 1995;15(6):983-7. PMID: 7581102
31. Lanternier F, Dannaoui E, Morizot G, Elie C, Garcia-Hermoso $D$, Huerre $M$, et al. A global analysis of mucormycosis in France: the RetroZygo Study (2005-2007). Clin Infect Dis. 2012;54 Suppl 1:S35-43. PMID:22247443

32. Ojeda-Uribe $M$, Herbrecht $R$, Kiefer MH, Schultz $P$, Chain J, Chenard MP, et al. Lessons from a case of oromandibular mucormycosis treated with surgery and a combination of amphotericin B lipid formulation plus caspofungin. Acta Haematol. 2010;124(2):98102. PMID:20689269

33. Spellberg B, Edwards J, Jr., Ibrahim A. Novel perspectives on mucormycosis: pathophysiology, presentation, and management. Clin Microbiol Rev. 2005;18(3):556-69. PMID:16020690

34. Reed C, Bryant R, Ibrahim AS, Edwards J, Jr., Filler SG, Goldberg $R$, et al. Combination polyene-caspofungin treatment of rhino-orbital-cerebral mucormycosis. Clin Infect Dis. 2008;47(3):364-71. PMID:18558882

35. Spellberg $B$, Ibrahim A, Roilides $E_{1}$ Lewis RE, Lortholary O, Petrikkos $G$, et al. Combination therapy for mucormycosis: why, what, and how? Clin Infect Dis. 2012;54 Suppl 1:S73-8. PMID: 22247449 